\title{
Modulation of Autoimmune Demyelination by Laquinimod via Induction of Brain-Derived Neurotrophic Factor
}

\author{
Jan Thöne, * Gisa Ellrichmann, * Silvia Seubert, ${ }^{*}$ \\ Isabella Peruga, ${ }^{*}$ De-Hyung Lee, ${ }^{*}$ \\ Rebecca Conrad, ${ }^{\dagger}$ Liat Hayardeny, ${ }^{\ddagger}$ \\ Giancarlo Comi, ${ }^{\S}$ Stefan Wiese, ${ }^{\dagger}$ Ralf A. Linker, ${ }^{*}$ \\ and Ralf Gold*

\begin{abstract}
From the Department of Neurology at St. Josef Hospital * and the Department of Cell Morphology and Molecular Neurobiology, ${ }^{\dagger}$ Group for Molecular Cell Biology, Rubr-University Bochum, Bochum, Germany; Teva-Pharmaceutical Industries Ltd., ${ }^{\neq}$ Netanya, Israel; and the Department of Neurology, Institute of Experimental Neurology, University Vita-Salute, Scientific
\end{abstract} \\ Institute San Raffaele, Milan, Italy
}

Laquinimod is a promising, orally available compound that has been successfully evaluated in placebo-controlled phase II/III studies of relapsing-remitting multiple sclerosis (MS). Studies are ongoing to further define laquinimod's modulatory mechanisms. Analyses in the animal model of experimental autoimmune encephalomyelitis (EAE) demonstrate that laquinimod reduces infiltration of leukocytes into the central nervous system, induces a Th1 to Th2/3 shift, and suppresses Th17 responses. To evaluate the potential neuroprotective capacity of laquinimod via modulation of brain-derived neurotrophic factor (BDNF), we analyzed the expression of BDNF in blood samples from 203 MS patients treated with laquinimod. Furthermore, we investigated the effect of laquinimod in EAE using a conditional BDNF knockout strain lacking BDNF expression in myeloid cells and $T$ cells (LLF mice). Treatment with laquinimod resulted in a significant and persistent increase in BDNF serum levels of MS patients when compared to baseline and placebotreated patients. LLF mice treated with laquinimod display a more severe EAE disease course in comparison to wild-type mice. Furthermore, laquinimod-treated wild-type monocytes secreted an anti-inflammatory cytokine pattern in comparison to untreated wildtype monocytes and treated LLF monocytes. Adoptive transfer of laquinimod stimulated monocytes into mice with EAE ameliorated the disease course. Consistent with immunomodulatory properties, laquinimod skewed monocytes toward a regulatory phenotype and also acted via modulation of BDNF, which may contribute to neuroprotection in MS patients. (Am J Pathol 2012, 180:267-274; DOI: 10.1016/j.ajpath.2011.09.037)

Laquinimod (ABR-215062) is an orally available synthetic compound designed for the treatment of relapsing-remitting multiple sclerosis (RRMS) with linomide (roquinimex) as the lead compound. In contrast to linomide, whose commercial launch was stopped as a result of severe side effects, laquinimod shows a much improved riskbenefit profile in clinical trials. ${ }^{1}$ It was successfully evaluated in placebo-controlled phase $\mathrm{I} / \mathrm{ll} \mathrm{b}$ studies and is currently under investigation in two global phase III clinical trials (ALLEGRO study; http://www.clinicaltrials.gov identifier NCT00509145, BRAVO study http://www. clinicaltrials.gov identifier NCT00605215). ${ }^{1}$ However, its mechanism of action is not yet fully elucidated. Studies conducted in the mouse model of multiple sclerosis (MS), experimental autoimmune encephalomyelitis (EAE), demonstrated a reduced infiltration of leukocytes into the central nervous system (CNS), a Th1 to Th2/3 shift, and possibly also suppression of proinflammatory Th17 responses. ${ }^{2-4}$ Additionally, studies in a rat model of experimental autoimmune neuritis showed milder regional de-

Supported by a grant from Teva Pharmaceuticals Industries Ltd., Netanya, Israel.

Accepted for publication September 20, 2011.

R.A.L. and R.G. share senior authorship.

Disclosures: Serum samples from the LAQ/5062 clinical trial and laquinimod were provided by Teva Pharmaceuticals Industries Ltd., Netanya, Israel. G.C. is head of the laquinimod clinical steering committee; R.G. is a member of the data safety monitoring board. L.H. is the Global Scientific Director GIP of Teva Pharmaceuticals Industries Ltd.

Supplemental material for this article can be found at $h$ ttp://ajp. amjpathol.org or at doi: 10.1016/j.ajpath.2011.09.037.

Address reprint requests to Jan Thöne, M.D., Department of Neurology at St. Josef-Hospital Bochum, Ruhr-University Bochum, Gudrunstr. 56, 44791 Bochum, Germany. E-mail: jan_thoene@gmx.de. 
myelination within the peripheral nervous system after treatment with laquinimod. ${ }^{5}$

Neurotrophins, such as brain-derived neurotrophic factor (BDNF), are essential for the development and maintenance of neurons and axons in the CNS. Although BDNF is mainly produced by neurons, several types of immune cells, including $T$ cells (TC), B cells, and monocytes, also secrete BDNF. ${ }^{6}$ Production and release of BDNF by infiltrating immune cells within the CNS may therefore contribute to the repair or survival of neurons, thus limiting brain damage. Interestingly, peripheral blood mononuclear cells of untreated RRMS patients secrete significantly lower levels of BDNF, but secretion is restored to the levels of healthy control animals by immunomodulatory treatment with drugs such as glatiramer acetate. ${ }^{7-11}$ In addition, recent data revealed a functional role of BDNF in autoimmune demyelination by mediating axon protection. ${ }^{12}$

To further elucidate the mechanism of action of laquinimod and to examine its potential neuroprotective capacity, we evaluated the levels of BDNF in the serum of RRMS patients treated with laquinimod in phase II clinical trials. Additionally, we investigated the effects of laquinimod in a conditional knockout model with a deficiency of BDNF in myeloid cells and TC. ${ }^{12}$ Last, we examined the influence of laquinimod on monocytes in EAE. Our data show that laquinimod ameliorates EAE disease course via BDNF-dependent mechanisms and leads to a sustained modulation of CD11bpositive monocytes toward a regulatory phenotype.

\section{Materials and Methods}

\section{Subjects and BDNF Analyses}

Serum samples from 203 RRMS patients ( $n=99$ in the placebo group and $n=104$ in the laquinimod group treated with $0.6 \mathrm{mg}$ per day) participating in the phase II laquinimod study 5062 were evaluated in a blinded fashion at three different time points for the presence of BDNF with a highly specific sandwich enzyme-linked immunosorbent assay (ELISA) without cross-reactivity or interference to beta-nerve growth factor, glial derived neurotrophic factor, neurotrophin-3 or neurotrophin-4 (R\&D Systems, Minneapolis, MN). Further, neurotrophin-3 and a global panel of 16 other cytokines/chemokines [IL-1 $\beta$, IL-2, IL-4, IL-5, IL-6, IL-8, IL-10, IL-12p40, IL-12p70, IL$15, \mathrm{IL}-17$, macrophage inflammatory proteins $1 \mathrm{a}$ and $1 \mathrm{~b}$, macrophage chemoattractant protein-1, tumor necrosis factor (TNF)- $\alpha$, and interferon (IFN)- $\gamma$ ] were analyzed with a highly specific sandwich ELISA (R\&D Systems). ${ }^{13} \mathrm{Ad}-$ ditionally, we correlated clinical and neuroimaging parameters, including expanded disability status scale, total number of T1-enhancing lesions (change from baseline), total number of new T2 lesions (change from baseline), total number of new $\mathrm{T} 1$ hypointense lesions (change from baseline), and T2 lesion volume to serum BDNF levels.

The protocol was approved by the institutional review boards of the participating centers. Patients provided written informed consent before undergoing any procedures. All experiments were performed in accordance with the
Code of Ethics of the World Association (Declaration of Helsinki).

\section{Dorsal Root Ganglion Neuronal Survival Assay}

Embryonic mouse dorsal root ganglion neurons were isolated as described previously. ${ }^{12,14}$ Selection of samples ( $n=7$ per group) reflected the mean BDNF difference between placebo and laquinimod obtained by ELISA. Initial counting of plated cells was performed when all cells were adherent after 4 hours of incubation. Surviving phase bright cells were counted after 24 hours of incubation with patient serum at a dilution of 1:1000. Five visual fields ( $1.9 \mathrm{~mm}^{2}$ per field) were counted in each well at each time point.

\section{Mice}

C57BL/6 mice [wild-type (WT) mice] were obtained from Harlan Laboratories (Borchen, Germany). BDNF conditional knockout mice (LLF mice) were back-crossed onto the C57BL/6 background for more than 10 generations. LLF mice were bred at the in-house animal care facilities at the Ruhr-University Bochum, Germany, as described in detail previously. ${ }^{12}$ All mice were housed under pathogen-free conditions at the animal facility of the RuhrUniversity Bochum. All animals were handled in accordance with good animal practice as defined by the relevant national and local welfare bodies. All experiments were reviewed and approved by the North-RhineWestphalia authorities for animal experimentation.

\section{$E A E$}

EAE was induced in mice that were 8 to 10 weeks old with $200 \mu \mathrm{g}$ of myelin oligodendrocyte glycoprotein 35-55 (MOG ${ }_{35-55}$ ) peptide (Charite, Berlin, Germany) in an equal volume of complete Freund's adjuvant containing Mycobacterium tuberculosis H37RA (Difco, Detroit, MI). On days 0 and 2, a total of $200 \mathrm{ng}$ of pertussis toxin (List/Quadratec, Campbell, CA) was applied i.p. Animals were weighed and scored daily as described previously. ${ }^{15}$

\section{Isolation of $\mathrm{CD} 11 \mathrm{~b}^{+}$Cells and Adoptive Cell Transfer}

Splenic $\mathrm{CD} 11 \mathrm{~b}^{+}$cells were isolated by magnetic cell sorting according to the manufacturers' protocols (Miltenyi Biotec, Auburn, CA). The purification level of CD11 $\mathrm{b}^{+}$ cells was routinely $>95 \%$ (see Supplemental Figure S1 at http://ajp.amjpathol.org). The expression of major histocompatibility complex (MHC) class II (MHC-II), CD275 (ICOS-L), CD40, and co-stimulatory molecules such as CD80 and CD86 was evaluated before cell transfer. On day 9 of ongoing MOG-EAE, CD11 ${ }^{+}$cells were derived from either laquinimod [5 mg/kg body weight (bw)] treated WT mice, laquinimod (5 mg/kg bw)-treated LLF mice, or sham (PBS)-treated WT mice. CD11 $b^{+}$cells $(1 \times$ $10^{6}$ cells per mouse versus $5 \times 10^{6}$ cells per mouse) 
were intravenously transferred into recipients on day 10 of ongoing MOG-EAE.

\section{Isolation of $\mathrm{CD} 11 \mathrm{~b}^{+}$Monocytes and Co-Culture with MOG-Activated TC}

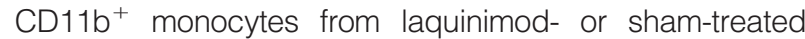
MOG-EAE mice were isolated as described above. We co-cultured monocytes with MOG activated TC from WT mice. We evaluated the expression of Th1 and Th2 cytokines by ELISA and the frequency of $\mathrm{CD} 4^{+} \mathrm{CD} 25^{+} \mathrm{FoxP}^{+}$ regulatory $\mathrm{TC}$ (Treg) by fluorescence-activated cell sorting (FACS) staining (eBioscience, San Diego, CA).

\section{Antibodies and Flow Cytometric Analysis}

FACS analyses were performed with a FACS Canto II (BD Pharmingen, Heidelberg, Germany) machine and CellQuest software. Monoclonal antibodies purchased from $\mathrm{BD}$ Pharmingen were used to detect CD3, CD4, CD8, CD11b, CD11c, CD80, CD86, CD275, MHC-II, FoxP3, and annexin $\mathrm{V}$ in accordance with the manufacturers' instructions as described previously. ${ }^{16}$

\section{ELISA}

Cell culture supernatants were collected, formed into aliquots, and stored at $-20^{\circ} \mathrm{C}$. Immediately before measurement, aliquots were brought to room temperature and analyzed for cytokines [IL-10, TNF- $\alpha$, transforming growth factor (TGF)- $\beta$, macrophage chemoattractant protein-1, IL-1 $\alpha$ ] with sandwich ELISA kits (BD Pharmingen; R\&D Systems) according to manufacturer protocols.

\section{Immunohistochemistry}

Immunohistochemistry was performed on 5- $\mu \mathrm{m}$ paraffinembedded sections. If necessary, antigen unmasking was achieved. After inhibition of unspecific binding with $10 \%$ bovine serum albumin, sections were incubated overnight at $4^{\circ} \mathrm{C}$ with the appropriate primary antibody in $1 \%$ bovine serum albumin. Secondary antibodies were used as indicated below. After blocking of endogenous peroxidase with $\mathrm{H}_{2} \mathrm{O}_{2}$, the peroxidase-based ABC detection system (Vectastain; Vector Laboratories, Wertheim, Germany) was used with diaminobenzidine as the chromogenic substrate. Specificity of staining was confirmed by omitting the primary antibody as a negative control. TC were labeled by rat anti-CD3 (1:200; Serotec, Düsseldorf, Germany) and macrophages by rat anti-mouse Mac-3 (1:200; BD Pharmingen). Bielschowsky silver impregnation was used to assess axonal pathology.

\section{RNA Isolation and Real-Time PCR}

RNA was isolated from mouse spinal cord and spleen by the Trizol method (Invitrogen, Darmstadt, Germany) following the manufacturer's instructions. cDNA was obtained by reverse transcription using SuperScript II Reverse Transcriptase (Invitrogen). Real-time PCR am- plifications were performed with Platinum Quantitative PCR SuperMix uracil DNA glycosylase (Invitrogen) on an Applied Biosystems 7500 fast real-time PCR system (Applied Biosystems, Darmstadt, Germany). As endogenous control, $\beta$-actin was used. TaqMan gene expression assays (Applied Biosystems) were used to analyze the expression of BDNF. Quantification of $\beta$-actin was achieved with primers $\beta$-actin S2 (5'-ATTGCCGACAGGATGCAGAA-3'), $\beta$-actin AS2 (5'-GCTGATCCACATCTGCTGGAA-3'), and $\beta$-actin Son2 (5'-FAM-CAAGATCATTGCTCCTCCTGAGCGCA-TAMRA-3'). BDNF mRNA expression was measured with mBDNF $S$ (5'-GGGCCGGATGCTTCCTT-3'), mBDNF AS (5'-GCAACCGAAGTATGAAATAACCATAG-3'), and mBDNF Son (5'-TTCCACCAGGTGAGAAGAGTGATGACCAT-3') as primers.

\section{Statistical Analysis}

Statistical analyses were performed by Prism software (GraphPad, San Diego, CA). Data are provided as mean \pm SEM. Significances between groups were examined by the Mann-Whitney U-test. Other statistical analyses were performed by repeated-measures analysis of variance for multiple comparisons. In all experiments, a $P$ value of $<0.05$ was defined as statistically significant, and $P<0.01$ was considered as highly statistically significant.

\section{Results}

\section{Effect of Laquinimod on BDNF Serum Levels in Patients with RRMS}

Laquinimod (0.6 mg per day) resulted in a significant and specific increase in BDNF serum levels compared to baseline and placebo treatment after 12 and 36 weeks of treatment (Figure 1, A-C). In total, a significant and robust BDNF increase occurred in $76 \%$ of laquinimodtreated patients. In individual patients, an up to 11-fold increase in BDNF serum levels was observed (Table 1). In contrast, neither neurotrophin-3 nor any other cytokine/ chemokine level differed significantly between groups. Bioactivity of serum BDNF was demonstrated in an in vitro dorsal root ganglion survival assay. Blinded quantification of surviving neurons 24 hours after addition of serum from selected representative participants in the LAQ/ 5062 trial revealed a significantly increased survival of neurons in the presence of sera from laquinimod-treated patients (Figure 1D).

Further subanalyses revealed no significant correlation between serum BDNF levels and magnetic resonance imaging or clinical parameters in this rather small cohort of patients as a result of the design of a phase Ilb MS study. Importantly, BDNF elevation in individual RRMS patients was independent from relapse rate. Moreover, there was no correlation between BDNF levels and age, sex, or baseline disability at any time point analyzed. Yet the source of serum BDNF after treatment with laquinimod remains questionable. Thus, further experiments using the EAE model were designed to explore the func- 


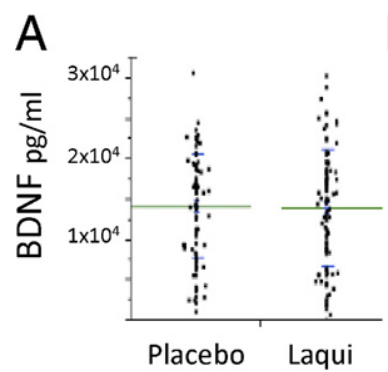

\section{B}

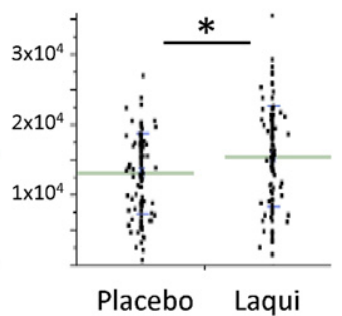

C

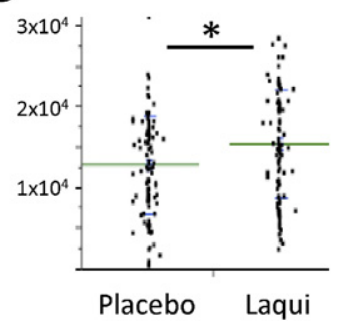

$\mathrm{D}$

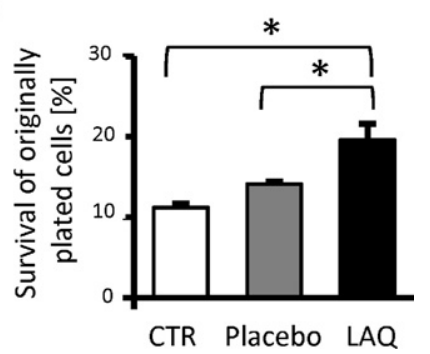

Figure 1. Laquinimod ( $0.6 \mathrm{mg}$ per day) modulates serum BDNF levels in RRMS. A-C: Laquinimod significantly increases BDNF levels in the serum of patients with RRMS after $12(\mathbf{B})$ and 36 weeks of treatment $(\mathbf{C})$ in comparison to baseline (A). D: Sera from laquinimod-treated patients with higher BDNF levels led to significantly higher numbers of surviving neurons compared to the addition of sera from placebo-treated patients. All data are shown as mean \pm SEM. ${ }^{*} P<0.05$ (repeated-measures analysis of variance).

tional role of BDNF for the immunomodulatory/protective effects of laquinimod in more detail.

\section{Functional Role of BDNF for the Therapeutic Effect of Laquinimod}

Mice with a conditional deficiency for BDNF in immune cells (LLF mice) and WT control animals were treated with laquinimod (5 mg/kg bw per day) starting at day 1 after MOG peptide immunization. ${ }^{12}$ Treatment with laquinimod resulted in a significant reduction of EAE incidence and disease severity in WT mice compared to sham-treated control animals (Figure 2, A and B). The beneficial effect of laquinimod was significantly attenuated in LLF mice (Figure 2, A and B). In parallel, real-time PCR analysis of spinal cord at day 20 after immunization demonstrated a significant up-regulation of BDNF mRNA in WT mice treated with laquinimod compared to untreated WT mice. Although this up-regulation almost reached BDNF levels of naive WT mice, LLF mice did not exhibit this effect (Figure 2C).

Real-time PCR analysis of spleen, also at day 20 after immunization, demonstrated a trend toward up-regulation of BDNF in WT mice treated with laquinimod compared to untreated WT mice, although this effect failed to reach statistical significance (Figure 2F). In WT spleen, laquinimod treatment led to a significant up-regulation of TGF- $\beta$ and IL-10 compared to sham-treated WT mice (Figure 2, G and $H)$. This increase was not present in the setting of selective BDNF deficiency in immune cells from LLF mice (Figure 2, $\mathrm{F}-\mathrm{H}$ ) or in the spinal cord (Figure 2, D and E).

We were next interested whether the BDNF dependent efficacy of laquinimod was sustained in a setting with more severe tissue damage. Therefore, we performed a histological analysis of mice with EAE after treatment with a suboptimal dose of laquinimod $(0.75 \mathrm{mg} / \mathrm{kg}$ bw per day). WT mice treated with a suboptimal dose of laquini- mod demonstrated a significant reduction of the inflammatory area and level of demyelination (Figure 3). Importantly, WT mice treated with a suboptimal laquinimod dose displayed a clear reduction of macrophage infiltration and a significant preservation of axonal densities in comparison to laquinimod-treated LLF mice and control animals (Figure $3 \mathrm{C}$ ). In contrast, the reduction of TC invasion reached only trend level in both WT and LLF laquinimod-treated mice (Figure 3C). In summary, our data suggest a BDNF dependent mechanism of action for laquinimod in autoimmune demyelination.

\section{Treatment with Laquinimod Targets Monocytes}

Our data in the MOG-EAE model indicate that laquinimod at a suboptimal dosage particularly targets cells of the monocyte lineage, while effects on TC are less pronounced. To further analyze the effect of laquinimod on monocytes and other immune cells in more detail, we examined the influence of laquinimod on the absolute number of different cell subsets.

We observed a significant reduction in the number of spleen-derived $\mathrm{CD} 11 \mathrm{~b}^{+}$monocytes after 9 days (before symptom onset) and 20 days of treatment with laquinimod ( $5 \mathrm{mg} / \mathrm{kg}$ bw per day) (Figure 4A). In contrast, analysis of the frequency of the absolute TC number as well as $\mathrm{CD}^{+}, \mathrm{CD}^{+}$, Treg, and $\mathrm{CD} 11 \mathrm{c}^{+} / \mathrm{B}_{2} 2 \mathrm{O}^{+}$plasmacytoid dendritic cells did not reveal any highly significant effects after laquinimod therapy (see Supplemental Figure S2A at $h$ ttp://ajp.amjpathol.org). Furthermore, we identified an increased expression of CD86 and a decrease of CD80, co-stimulatory molecules involved in either Th2 or Th1 response (Figure 4B). ${ }^{17,18}$

In LLF mice, the effect on the absolute monocyte cell number was obvious as well, yet the effect on the expression of co-stimulatory molecules was weaker (see Sup-

Table 1. BDNF Serum Concentration

\begin{tabular}{lcccc}
\hline Treatment & Baseline $(\mathrm{pg} / \mathrm{mL})$ & Month 3 $(\mathrm{pg} / \mathrm{mL})$ & Month 9 $(\mathrm{pg} / \mathrm{mL})$ & $P^{*}$ \\
\hline Placebo & $13662.55 \pm 6561.06$ & $12667.06 \pm 5821.95$ & $12627.28 \pm 6219.03$ & $<0.05$ \\
Laquinimod & $14212.89 \pm 7239.11$ & $15818.16 \pm 7135.58$ & $15487.05 \pm 6687.60$ & $<0.05$ \\
\hline
\end{tabular}

Laquinimod $0.6 \mathrm{mg} /$ day significantly increased BDNF serum concentration after 12 weeks and 36 weeks of treatment in comparison to baseline, while in the placebo group, no significant effects on BDNF levels were detectable. Data are presented as mean \pm SD.

${ }^{*}$ Repeated-measure analysis of variance. 
A
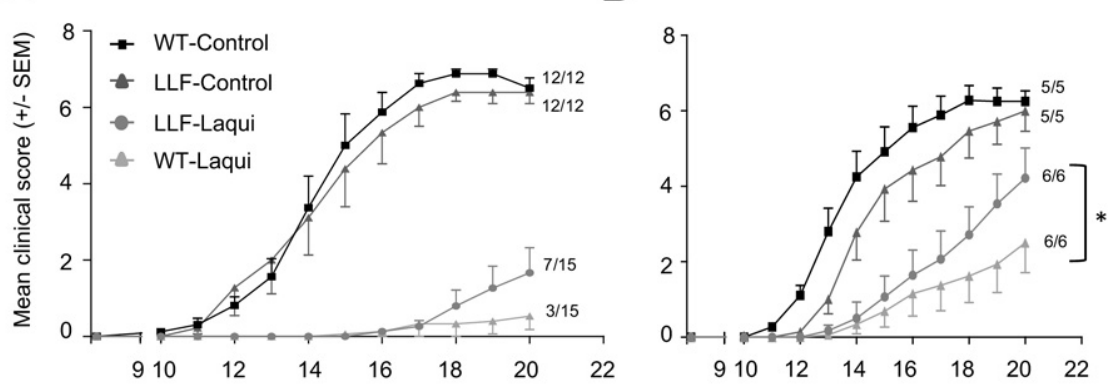

Days after immunization
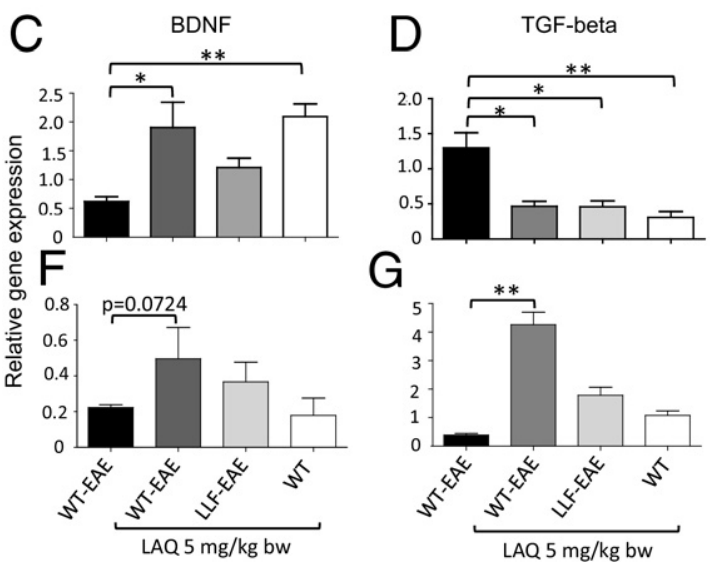

$\mathrm{G}$

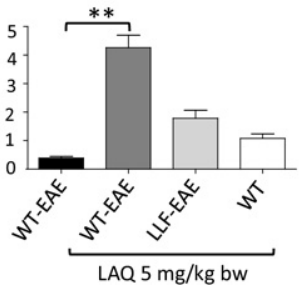

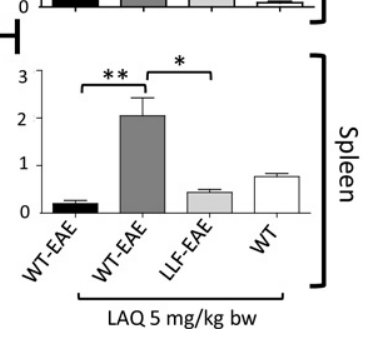

Figure 2. Laquinimod ameliorates EAE disease course via BDNF. A: WT mice treated with laquinimod ( $5 \mathrm{mg} / \mathrm{kg}$ bw daily) show an ameliorated EAE disease course in comparison to sham-treated control animals and laquinimodtreated LLF mice. Data are representative of three independent experiments with the same result. B: WT mice treated with a suboptimal dose of laquinimod $(0.75 \mathrm{mg} / \mathrm{kg}$ bw daily) display an ameliorated disease course in comparison to sham-treated control animals and laquinimod-treated LLF mice. Data are representative of three independent experiments with the same result. C-E: Real-time PCR analysis of spinal cord at day 20 after immunization reveals an increase in BDNF expression after laquinimod treatment $(5 \mathrm{mg} / \mathrm{kg}$ bw daily). Data are representative of three different experiments with the same result. F-H: Real-time PCR analyses taken from spleen at day 20 after immunization reveals a nonsignificant increase in BDNF and a significant increase of TGF- $\beta$ and IL-10 expression after laquinimod treatment $(5 \mathrm{mg} / \mathrm{kg}$ bw daily). Data are representative of three different experiments with the same result. All data are shown as mean \pm SEM. ${ }^{*} P<0.05,{ }^{* * *} P<0.01$ (repeatedmeasures analysis of variance). Numbers in the EAE graphs indicate the number of sick animals per total number of mice per group.

plemental Figure S2B at $h$ ttp://ajp.amjpathol.org). Finally, FACS analyses did not reveal an increase of the apoptosis marker annexin V in CD11 $b^{+}$cells (see Supplemental Figure S2C at http://ajp.amjpathol.org).

\section{Laquinimod Skews Monocytes to a Regulatory Type II Phenotype with the Potential to Suppress Th1 Responses}

To investigate whether laquinimod-treated monocytes mediate immune modulation in vivo, we adoptively trans-

\section{.}

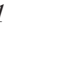


A

B
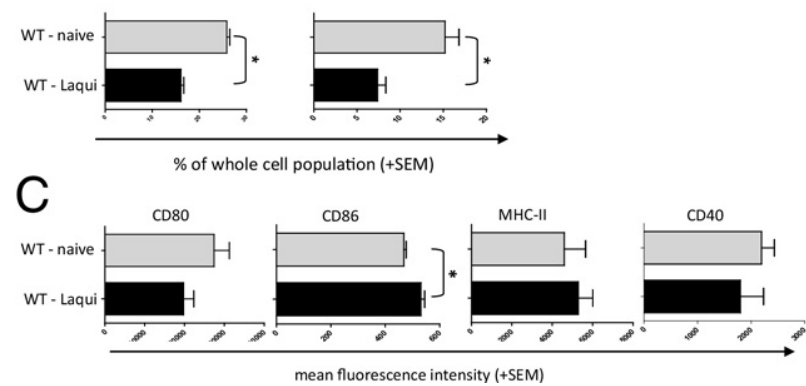

Figure 4. Laquinimod targets monocytes. A, B: FACS analysis showed a significantly reduced number of spleen-derived CD $11 \mathrm{~b}^{+}$monocytes from laquinimod $(5 \mathrm{mg} / \mathrm{kg} \mathrm{bw})$ treated WT mice in comparison to placebo treated WT mice after 9 days (A) and 20 days (B) of daily treatment. C: Laquinimodtreated monocytes showed a nonsignificant decrease of CD80 and a significant increase of CD86, while no effect on MHC-II and CD40 was detectable. Data are representative of three different experiments with the same result All data are shown as mean \pm SEM. ${ }^{*} P<0.05$.

laquinimod (5 mg/kg bw) treated MOG-WT mice secreted significantly more IL-10 and less IL-6 in comparison to sham-treated MOG immunized WT mice, whereas the secretion of IL- $1 \beta$, TNF- $\alpha$, and TGF- $\beta$ did not differ significantly between groups (Figure $5 \mathrm{~B}$ ).

To address whether laquinimod-treated monocytes isolated from MOG-EAE are able to direct TC deviation or promote development of Treg cells, we performed co-culture experiments with MOG activated TC. MOG activated TC co-cultured with sham-treated monocytes secreted significant amounts of the Th1 cytokine IFN- $\gamma$, while the addition of laquinimod-treated monocytes significantly suppressed IFN- $\gamma$ production (Figure $5 \mathrm{C}$ ). In contrast, the secretion of IL-4 and IL-10 did not significantly differ between cultures with addition of laquinimod- or sham-pretreated monocytes, and no expansion of Treg cells or influence on TC proliferation occurred (Figure 5C; see also Supplemental Figure S3A at http://ajp.amjpathol.org).

In a second set of experiments, we adoptively transferred CD11 $\mathrm{b}^{+}$cells $\left(5 \times 10^{6}\right)$ derived from laquinimod $(5 \mathrm{mg} / \mathrm{kg}$ bw)-treated MOG-immunized WT mice and MOG-immunized LLF mice into recipient WT mice with ongoing EAE versus CD11 $\mathrm{b}^{+}$cells derived from sham-treated MOG WT mice. Similar to Figure 5, adaptive transfer of laquinimodtreated $\mathrm{CD} 11 \mathrm{~b}^{+}$cells derived from MOG WT mice again significantly ameliorated the course of EAE (Figure 6A). In contrast, adaptive transfer of laquinimod-treated CD11 ${ }^{+}$ cells derived from LLF mice into recipient WT mice with ongoing EAE at early disease stage did not influence the disease course (Figure 6A). Histological analysis at the acute disease stage confirmed the clinical data and revealed a reduction of inflammatory infiltration as well as of demyelination (Figure 6B). Additionally, CD11b ${ }^{+}$cells derived from laquinimod-treated LLF mice secreted significantly less IL-10 compared to laquinimod-treated LLF mice (Figure 6C). Untreated CD11 ${ }^{+}$cells derived from MOGimmunized WT mice and MOG-immunized LLF mice, respectively, secreted similar levels of IL-10 (data not shown).

\section{Discussion}

In this study, we explore potential neuroprotective properties of the novel oral immunomodulator laquinimod by modulation of BDNF production. Our data indicate a direct and sustained effect of laquinimod on the up-regulation of bioactive BDNF in patients with RRMS. This finding was verified in the EAE model using a conditional BDNF knockout strain. Additionally, we demonstrate that laquinimod targets monocytes and skews the phagocyte population toward a regulatory phenotype, which in turn mediates immune modulation in vivo.

Studies including data from EAE models demonstrated the beneficial effect of directly administered BDNF or BDNF gene transfer for enhanced survival of injured neurons and regenerative capacity. ${ }^{19-22}$ Thus, pharmacological modulation of BDNF secretion or stimulation of BDNF transcription is a promising goal in MS. In contrast to licensed immunomodulatory therapies and pipeline drugs for MS, which primarily target the immune system, laquinimod may
A

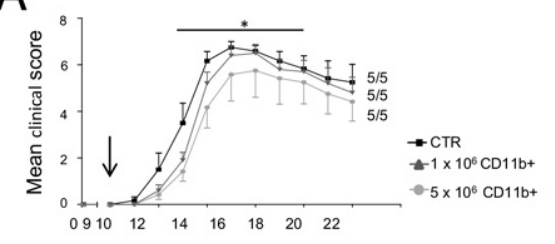

B

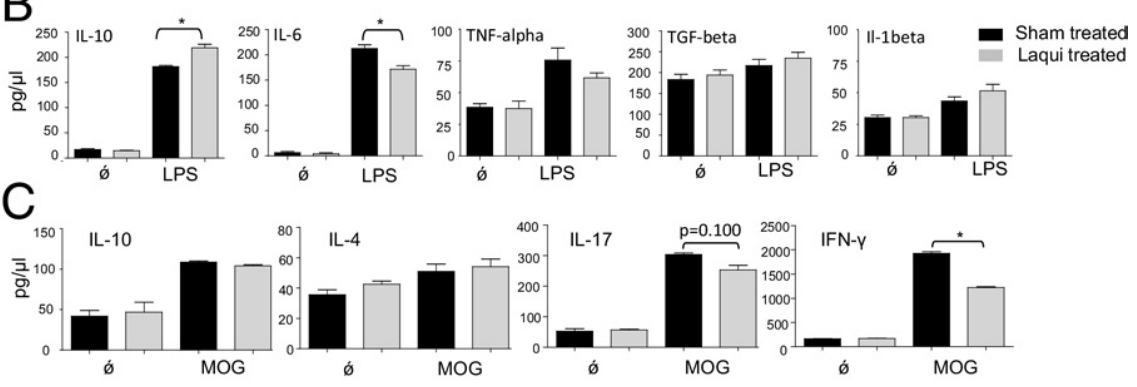

Figure 5. Laquinimod-treated monocytes have the capacity to modulate EAE. A: Transfer of $5 \times$ $10^{6}$ laquinimod-treated $\mathrm{CD}_{11 \mathrm{~b}^{+}}$cells ameliorates EAE disease course in comparison to transfer of $1 \times 10^{6}$ laquinimod-treated CD11b ${ }^{+}$cells and sham (PBS)-treated control animals. Numbers in the graph represent affected/total mice. Arrow indicates day of transfer. B: ELISA experiments demonstrate a significant increase of IL-10 and decrease of IL- 6 in laquinimod-treated $\mathrm{CD} 1 \mathrm{~b}^{+}$cells compared to sham-treated WT mice. Secretion of TNF- $\alpha$, TGF- $\beta$, or IL- $1 \beta$ did not differ significantly between groups. C: MOGactivated TC co-cultured with sham-treated monocytes show a significant increased secretion of IFN- $\gamma$ compared to laquinimod-treated monocytes. Secretion of IL-10 and IL-4 differ not significantly between groups. Data are representative of at least three independent experiments with the same result. All data are shown as mean \pm SEM. ${ }^{*} P<0.05$. 
A

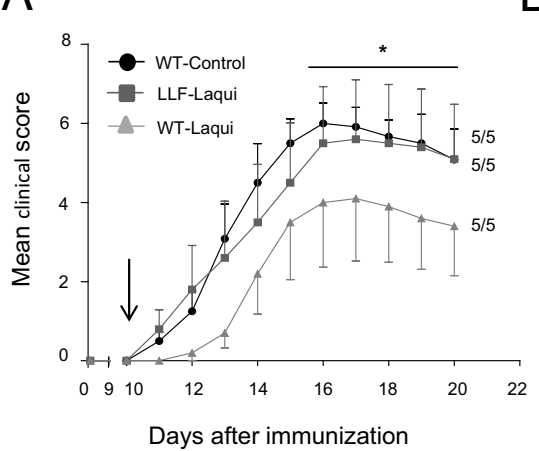

C
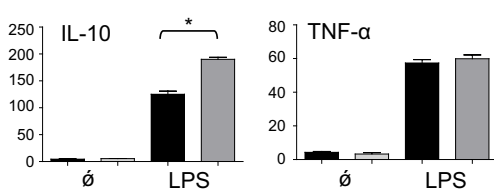

B
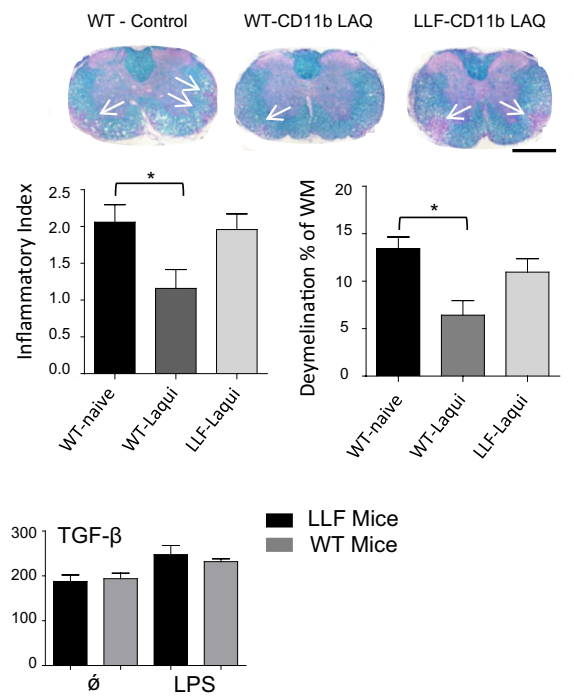

Figure 6. Effects of laquinimod on monocytes are BDNF dependent. A: Transfer of $5 \times 10^{6}$ laquinimod $(5 \mathrm{mg} / \mathrm{kg}$ body weight)-treated WT

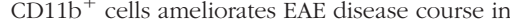
comparison to laquinimod-treated LLF CD11 $\mathrm{b}^{+}$ cells and untreated WT CD $11 b^{+}$cells. Numbers in the graph represent affected/total mice. Arrow indicates day of cell transfer. B: Histological analyses of $\mathbf{A}$. Mice treated with $5 \times 10^{6}$ laquinimod-treated $\mathrm{CD}_{11} \mathrm{~b}^{+}$cells show a significant reduction of both demyelination and inflammatory index compared to mice treated with laquinimod-treated LLF CD11b ${ }^{+}$cells and untreated WT CD $11 b^{+}$cells. Arrows mark demyelinated areas. Scale bar $=500 \mu \mathrm{m}$. C: ELISA experiments demonstrate a significant increase of IL-10 in laquinimod-treated WT CD $11 \mathrm{~b}^{+}$cells compared to laquinimod-treated LLF CD11b ${ }^{+}$cells. Secretion of TNF- $\alpha$ and TGF- $\beta$ did not differ significantly between groups. All data are shown as mean \pm SEM. ${ }^{*} P<0.05$. provide both immunomodulatory and neuroprotective mechanisms of action via BDNF-mediated pathways. ${ }^{23}$

The data on BDNF regulation in RRMS patients illustrate a robust and sustained increase of BDNF serum levels that do not depend on relapse rate reduction, a mechanism that has been shown to independently alter BDNF expression. ${ }^{24}$ Further, BDNF data are corroborated by dorsal root ganglion survival assay and EAE experiments. The beneficial efficacy of laquinimod is turned into a detrimental, rather disease-exacerbating action in mice deficient for BDNF in immune cells. WT mice treated with laquinimod show a significant preservation of axonal density in comparison to laquinimod-treated LLF mice and control animals, highlighting neuroprotective effects. In parallel, we observed an increased relative BDNF gene expression in both spinal cord and spleen in WT mice treated with laquinimod. In contrast, we found no significant correlation between any magnetic resonance imaging or clinical parameter to BDNF serum levels. However, neither duration nor sample size of this phase Ilb study was sufficiently powered to address these aspects. ${ }^{13}$ Furthermore, neither the imaging protocol of the LAQ/5062 trial nor its extension study (LAQ/5063) include magnetic resonance imaging techniques, eg, brain atrophy, as a marker for neuroprotection. ${ }^{13,25}$

Moreover, laquinimod targets cells of the monocyte lineage at the acute stage of MOG-EAE and leads to the generation of regulatory monocytes. In turn, this may result in a significant reduction of TC in early chronic MOG-EAE, as shown previously. ${ }^{4}$ To date, different stimuli that lead to the generation of regulatory monocytes have been identified and in particular include the immunomodulatory cytokine $\mathrm{IL}-10 .^{26}$ Moreover, regulatory monocytes themselves are characterized by an increased secretion of $\mathrm{LL}-10$ and thus induce immune regulation during $E A E{ }^{26}$ This may be particularly relevant for MS therapy as shown for glatiramer acetate, which is licensed as a disease-modifying drug for the treatment of RRMS. Glatiramer acetate induces the generation of regulatory monocytes with the capacity to reverse established EAE after intravenous transfer ${ }^{26}$ Similar to glatiramer acetate, laquinimod treatment promoted the devel- opment of regulatory monocytes characterized by an increased secretion of IL-10 and the capability to inhibit the secretion of the proinflammatory cytokine IFN- $\gamma$. This effect might result in both local immunomodulation within the CNS and immunomodulation in the peripheral immune system. Additionally, treatment with laquinimod reduced the number of splenic CD11 $\mathrm{b}^{+}$cells and induced an up-regulation of CD86 and down-regulation of CD80, co-stimulatory molecules usually expressed by regulatory cells. ${ }^{26}$ In summary, these findings underline the role of monocytes as effector cells in laquinimod mediated immune modulation.

Although adoptive transfer and EAE experiments in LLF mice demonstrated significant effects on EAE disease progression, both mechanisms of action do not fully explain the pronounced effect of laquinimod treatment on EAE. Thus, our data indicate a multifaceted mechanisms of action of laquinimod, including immunomodulatory and neuroprotective mechanisms of action that should be further tested in human specimens. Previous independent studies conducted in the EAE model already indicate reduced infiltration of leukocytes into the CNS, an altered cytokine balance in favor of a Th2/Th3 phenotype, and possible suppression of pro-inflammatory Th17 responses. ${ }^{2,4}$ Similar to these results, we detected a reduced infiltration of immune cells into the CNS and increased relative gene expression of splenic IL-10 and TGF- $\beta$ in WT mice treated with laquinimod compared to sham-treated control animals. Surprisingly, no effect on cytokine balance was obvious in laquinimod-treated LLF mice. Indeed, inflammatory cells, including monocytes, express BDNF and its receptor, trkB. ${ }^{27}$ Thus, BDNF might also participate in the activation and activity of monocytes and after secretion of pro-/anti-inflammatory cytokines. ${ }^{28,29}$ In line with this assumption, adaptive transfer of CD11 ${ }^{+}$cells taken from LLF mice failed to ameliorate EAE disease progression. Further, we found no IL-10 increase in laquinimod-treated CD11 ${ }^{+}$ cells taken from LLF mice.

In summary, our data imply at least a dual mode of action of laquinimod. On the one hand, laquinimod targets monocytes. On the other hand, laquinimod modu- 
lates BDNF, contributing to neuroprotective properties in $M S$ and EAE. We hypothesize that the increase of BDNF might also be involved in its effects on monocytes and subsequent increase of IL-10. In parallel, recent work elucidated the beneficial role of $\mathrm{IL}-10$ expression by monocyte-derived macrophages for neuroprotection; however, this point remains in question and ought to be addressed in more detail in further studies. ${ }^{30}$ To date, to our knowledge, selective targeting of monocytes has not been described for any other MS pipeline drug, highlighting an innovative mechanism of action of laquinimod.

\section{Acknowledgment}

We thank Bernadette Jesionek for her skillful technical assistance.

\section{References}

1. Thöne J, Gold R: Laquinimod: a promising oral medication for the treatment of relapsing-remitting multiple sclerosis. Expert Opin Drug Metab Toxicol 2011, 7:365-370

2. Yang JS, Xu LY, Xiao BG, Hedlund G, Link H: Laquinimod (ABR215062) suppresses the development of experimental autoimmune encephalitis, modulates the Th1/Th2 balance and induces the Th3 cytokine TGF-beta in Lewis rats. J Neuroimmunol 2004, 156:3-9

3. Brunmark CA, Runström A, Ohlsson L, Sparre B, Brodin T, Aström M, Hedlund G: The new orally active immunoregulator laquinimod (ABR215062) effectively inhibits development and relapses of experimental autoimmune encephalomyelitis. J Neuroimmunol 2002, 130:163-172

4. Wegner C, Stadelmann C, Pförtner R, Raymond E, Feigelson S, Alon R, Timan B, Hayardeny L, Brück W: Laquinimod interferes with migratory capacity of $\mathrm{T}$ cells and reduces $\mathrm{IL}-17$ levels, inflammatory demylination and acute axonal damage in mice with experimental autoimmune encephalomyelitis. J Neuroimmunol 2010, 227:133-143

5. Zou LP, Abbas N, Volkmann I, Nennesmo I, Levi M, Wahren B, Winblad B, Hedlund G, Zhu J: Suppression of experimental autoimmune neuritis by ABR-215062 is associated with altered Th1/Th2 balance and inhibited migration of inflammatory cells into the peripheral nerve tissue. Neuropharmacology 2002, 42:731-739

6. Kerschensteiner M, Gallmeier E, Behrens L, Leal L, Misgeld T, Klinkert WE, Kolbeck R, Hoppe E, Oropeza-Wekerle RL, Bartke I, Stadelmann C, Lassmann H, Wekerle H, Hohlfeld R: Activated human T cells. B cells, and monocytes produce brain-derived neurotrophic factor in vitro and in inflammatory brain lesions: a neuroprotective role of inflammation? J Exp Med 1999, 189:865-887

7. Azoulay D, Urshansky N, Karni A: Low and dysregulated BDNF secretion from immune cells of MS patients is related to reduced neuroprotection. J Neuroimmunol 2008, 195:186-193

8. De Santi L, Annunziata P, Sessa E, Bramanti P: Brain-derived neurotrophic factor and TrkB receptor in experimental autoimmune encephalomyelitis and multiple sclerosis. J Neurol Sci 2009, 287:17-26

9. Aharoni R, Eilam R, Domev H, Labunskay G, Sela M, Arnon R: The immunomodulator glatiramer acetate augments the expression of neurotrophic factors in brains of experimental autoimmune encephalomyelitis mice. Proc Natl Acad Sci USA 2005, 102:19045-19050

10. Ziemssen T, Kümpfel T, Klinkert WE, Neuhaus O, Hohlfeld R: Glatiramer acetate-specific T-helper 1- and 2-type cell lines produce BDNF: implications for multiple sclerosis therapy. Brain derived neurotrophic factor. Brain 2002, 125:2381-2391

11. Linker RA, Gold R, Lühder F: Function of neurotrophic factors beyond the nervous system: inflammation and autoimmune demyelination. Crit Rev Immunol 2009, 29:43-68

12. Linker RA, Lee DH, Demir S, Wiese S, Kruse N, Siglienti I, Gerhardt E, Neumann H, Sendtner M, Lühder F, Gold R: Functional role of brainderived neurotrophic factor in neuroprotective autoimmunity: thera- peutic implications in a model of multiple sclerosis. Brain 2010, 133:2248-2263

13. Comi G, Pulizzi A, Rovaris M, Abramsky O, Arbizu T, Boiko A, Gold R, Havrdova E, Komoly S, Selmaj K, Sharrack B, Filippi M, LAQ/5062 Study Group: Effect of laquinimod on MRI-monitored disease activity in patients with relapsing-remitting multiple sclerosis: a multicentre, randomised, double-blind, placebo-controlled phase Ilb study. Lancet 2008, 371:2085-2092

14. Wiese S, Herrmann T, Drepper C, Jablonka S, Funk N, Klausmeyer A, Rogers ML, Rush R, Sendtner M: Isolation and enrichment of embryonic mouse motoneurons from the lumbar spinal cord of individual mouse embryos. Nat Protoc 2010, 5:31-38

15. Linker RA, Mäurer M, Gaupp S, Martini R, Holtmann B, Giess R, Rieckmann P, Lassmann H, Toyka KV, Sendtner M, Gold R: CNTF is a major protective factor in demyelinating CNS disease: a neurotrophic cytokine as modulator in neuroinflammation. Nat Med 2002, 8:620-624

16. Stegbauer J, Lee DH, Seubert S, Ellrichmann G, Manzel A, Kvakan H, Muller DN, Gaupp S, Rump LC, Gold R, Linker RA: Role of the renin-angiotensin system in autoimmune inflammation of the central nervous system. Proc Natl Acad Sci USA 2009, 106:14942-14947

17. Kuchroo VK, Das MP, Brown JA, Ranger AM, Zamvil SS, Sobel RA, Weiner HL, Nabavi N, Glimcher LH: B7-1 and B7-2 costimulatory molecules activate differentially the Th1/Th2 developmental pathways: application to autoimmune disease therapy. Cell 1995 , 80:707-718

18. Ranger AM, Das MP, Kuchroo VK, Glimcher LH: B7-2 (CD86) is essential for the development of IL-4-producing $T$ cells. Int Immunol 1996, 8:1549-1560

19. Sendtner M, Holtmann B, Kolbeck R, Thoenen H, Barde YA: Brainderived neurotrophic factor prevents the death of motoneurons in newborn rats after nerve section. Nature 1992, 360:757-759

20. Yan Q, Elliott J, Snider WD: Brain-derived neurotrophic factor rescues spinal motor neurons from axotomy-induced cell death. Nature 1992 , 360:753-755

21. Gravel C, Götz R, Lorrain A, Sendtner M: Adenoviral gene transfer of ciliary neurotrophic factor and brain-derived neurotrophic factor leads to long-term survival of axotomized motor neurons. Nat Med 1997, 3:765-769

22. McTigue DM, Horner PJ, Stokes BT, Gage FH: Neurotrophin-3 and brain-derived neurotrophic factor induce oligodendrocyte proliferation and myelination of regenerating axons in the contused adult rat spinal cord. J Neurosci 1998, 18:5354-5365

23. Linker RA, Kieseier BC, Gold R: Identification and development of new therapeutics in multiple sclerosis. Trends Pharmacol Sci 2008, 29:558-565

24. Comi G, Abramsky O, Arbizu T, Boyko A, Gold R, Havrdová E, Komoly S, Selmaj K, Sharrack B, Filippi M, LAQ/5063 Study Group: Oral laquinimod in patients with relapsing-remitting multiple sclerosis: 36-week double-blind active extension of the multi-centre, randomized, double-blind, parallel-group placebo-controlled study. Mult Scler 2010, 16:1360-1366

25. Caggiula M, Batocchi AP, Frisullo G, Angelucci F, Patanella AK, SancriccaC, Nociti V, Tonali PA, Mirabella M: Neurotrophic factors and clinical recovery in relapsing-remitting multiple sclerosis. Scand $\mathrm{J}$ Immunol 2005, 62:176-182

26. Weber MS, Prod'homme T, Youssef S, Dunn SE, Rundle CD, Lee L, Patarroyo JC, Stüve O, Sobel RA, Steinman L, Zamvil SS: Type ॥ monocytes modulate $T$ cell-mediated central nervous system autoimmune disease. Nat Med 2007, 13:935-943

27. Vega JA, Garcia-Suarez O, Hannestad J, Perez-Perez M, Germana A: Neurotrophins and the immune system. J Anat 2003, 203:1-19

28. Wen AY, Sakamoto KM, Miller LS: The role of the transcription factor CREB in immune function. J Immunol 2010, 185:6413-6419

29. Sánchez-Muñoz I, Sánchez-Franco F, Vallejo M, Fernández A, Palacios N, Fernández M, Sánchez-Grande M, Cacicedo L: Regulation of somatostatin gene expression by brain derived neurotrophic factor in fetal rat cerebrocortical cells. Brain Res 2011, 1375:28-40

30. London A, Itskovich E, Benhar I, Kalchenko V, Mack M, Jung S, Schwartz M: Neuroprotection and progenitor cell renewal in the injured adult murine retina requires healing monocyte-derived macrophages. J Exp Med 2011, 208:23-39 